\title{
Double minute chromosomes harboring MDM2 amplification in a pediatric atypical lipomatous tumor
}

\begin{tabular}{|r|l|}
\hline Journal: & Genes, Chromosomes and Cancer \\
\hline Manuscript ID & GCC-18-0279.R2 \\
\hline Wiley - Manuscript type: & Brief Report \\
\hline Keywords: & $\begin{array}{l}\text { Atypical lipomatous tumor, pediatric, double minute chromosome, well } \\
\text { differentiated liposarcoma, dedifferentiated liposarcoma }\end{array}$ \\
\hline
\end{tabular}

\section{SCHOLARONE \\ Manuscripts}




\title{
Double minute chromosomes harboring MDM2 amplification in a pediatric atypical lipomatous tumor
}

\author{
Bérengère Dadone-Montaudié ${ }^{1,2}$, Fanny Burel-Vandenbos ${ }^{2}$, Christine Soler ${ }^{3}$, Olivier \\ Rosello $^{4}$, Corinne Boyer ${ }^{5}$, Thibault Fabas ${ }^{1}$, Laurence Bianchini ${ }^{1}$, Florence Pedeutour ${ }^{1}$ \\ ${ }^{1}$ Laboratory of Solid Tumors Genetics, Institute for Research on Cancer and Aging of Nice \\ (IRCAN) CNRS UMR 7284/INSERM U1081, Université Côte d'Azur (UCA), Centre \\ Hospitalier Universitaire de Nice, Faculté de Médecine, 28 avenue de Valombrose 06000 Nice, \\ France
}

${ }^{2}$ Central Laboratory of Pathology, Nice University Hospital, Hôpital Pasteur, 30 avenue de la Voie Romaine 06000 Nice, France

${ }^{3}$ Department of Pediatric Onco-hematology, Nice University Hospital, Hôpital Archet 2, 151 route de Saint-Antoine, 06200 Nice, France

${ }^{4}$ Department of Pediatric Surgery, Hôpital Lenval, 57 Avenue de la Californie, 06200 Nice, France

${ }^{5}$ Department of Pediatric Radiology, Hôpital Lenval, 57 Avenue de la Californie, 06200 Nice, France

Corresponding author: Florence Pedeutour, Laboratoire de Génétique des Tumeurs Solides, Faculté de Médecine, 28 avenue de Valombrose, 06000 Nice. Phone number: +33493377012 . Fax number: +33492037529 .

Email: florence.pedeutour@unice.fr

Funding: This work was supported by the Institut National du Cancer (INCa) and the Direction Générale de 1'Offre de Soins (DGOS) (PRT-K 2016, FILIPO), the Direction de la Recherche 
et de l'Innovation (DRCI) du Centre Hospitalier Universitaire de Nice (prime d'intéressement à la recherche), the Fondation ARC pour la recherche sur le cancer, the Alan B. Slifka Foundation, the GEMLUC (Groupement des Entreprises Monégasques dans la Lutte contre le Cancer) and Infosarcomes. BDM was a Fondation ARC pour la recherche sur le cancer fellowship recipient at the time of the study.

\begin{abstract}
Adipocytic tumors are rare in children and are mostly benign. Less than 25 cases of pediatric well-differentiated liposarcoma (WDLPS), atypical lipomatous tumors (ALT) and dedifferentiated liposarcoma (DDLPS) have been reported. Among them, only three cases were genetically analyzed. We describe the genetic features of a rapidly growing adipose tumor that occurred in the thigh of a 7-year-old girl. Histologically, it was composed of mature adipocytic cells with a few atypia. Molecular analysis showed high-level amplification of the 12q13-21 region including $M D M 2$ among 64 amplified genes. MDM2 amplification is a diagnostic hallmark of ALT/WDLPS/DDLPS. In adult cases it is typically located in ring or giant marker chromosomes. In the present case, extra-copies of $M D M 2$ were located on double minute chromosomes (dmin). This raised the hypothesis of dmin being precursors of adult's rings and giant markers and may provide indications for a better understanding of the mechanisms of adipose tumor oncogenesis.
\end{abstract}

\title{
Keywords
}

Atypical lipomatous tumor; well-differentiated liposarcoma; dedifferentiated liposarcoma; pediatric; double minute chromosome 


\section{INTRODUCTION}

The epidemiologic features of adipose tumors show differences between children and adults. Adipocytic tumors are very uncommon in children. ${ }^{1}$ They are mainly represented by two benign entities: lipoblastoma, which is almost exclusively a pediatric tumor, and lipoma, one of the most frequent tumors of adulthood. Malignant pediatric adipose tumors are even rarer, accounting for $2 \%$ of all soft tissue sarcomas in patients younger than 20 years old. ${ }^{1}$ Myxoid liposarcoma is the most frequent pediatric liposarcoma, followed by pleomorphic liposarcoma. Well-differentiated liposarcoma (WDLPS) / atypical lipomatous tumors (ALT) / dedifferentiated liposarcoma (DDLPS) appear to be exceptional in children whereas they are the most frequent types of adult sarcomas. They usually occur in middle-aged adults, with a peak incidence in the sixth decade. ${ }^{1,2}$ To the best of our knowledge, less than 25 pediatric WDLPS/ALT/DDLPS cases have been reported so far. ${ }^{3-11}$ Adult WDLPS/ALT/DDLPS have been well characterized at the clinical and biological levels. Genetically, they share amplification of the MDM2 gene as a common recurrent and pathognomonic feature. In adult WDLPS/ALT/DDLPS, MDM2 amplification is typically located in supernumerary ring or giant rod marker chromosomes, together with several amplified genes from the 12q13-15 region. Additional genes originating from other chromosomal segments (inter-patient variability) are often co-amplified; as such they are part of the composition of the large supernumerary chromosomes. ${ }^{2}$ Whereas the genetic landscape of adult ALT/WDLPS/DDLPS is now well defined, the characteristics of its pediatric counterpart remain almost unexplored. Indeed, the status of MDM2 has been investigated in only three of the few reported pediatric cases. ${ }^{3-5}$

Here we report the first complete chromosomal and genetic description of a pediatric ALT that showed both similarities and differences in comparison to adult cases.

\section{MATERIALS AND METHODS}




\subsection{Case report}

A 7-year-old-girl presented with a rapidly growing, painless mass of the right thigh. Magnetic resonance imagering revealed a well-defined, heterogeneous, lipomatous tumor measuring 7x7x5cm located in adductor muscle (Fig. 1A). A biopsy was first performed. Two months later, following collegial discussions on potential functional consequences of a wide surgical procedure versus oncologic risks of insufficient tumor removal, it was followed by a complete surgical resection (Fig. 1B). No recurrence or metastasis was detected after six months of follow-up. Informed consent for molecular analyses from both parents and child has been obtained.

\subsection{Pathological, cytogenetic and immunohistochemical analyses}

Tumor samples from the surgical biopsy and the surgical excision were formalin-fixed and paraffin-embedded (FFPE) according to standard protocols. The tumor fragment obtained from the surgical excision was sampled in 17 blocks. In addition, a fresh fragment was used for mechanical and enzymatic disaggregation followed by short-term cell cultures ( 8 days), metaphase cells harvest and spreading according to standard cytogenetic procedures. Antigene retrieval and immunohistochemistry were done on FFPE tissue sections using the Agilent Dako Autostainer Link 48 (Les Ulis, France) and antibodies against MDM2 (1/25, pH6, BioSB, Santa Barbara, CA) and HMGA2 (1/100, pH9, BioCheck, San Francisco, CA).

\subsection{Fluorescence in situ hybridization (FISH) analysis}

FISH analyses were performed on interphase and metaphase cells from short-term culture and on FFPE tissue sections from both the biopsy and the surgical excision, using probes for MDM2 (green signal) and centromere 12 (red signal) (ZytoLight SPEC MDM2/CEN 12 Dual Color, ZytoVision, Bremerhaven, Germany) and PLAG1 (BACs RP11-242J1 (3 'PLAG1; green signal) and RP11-97K15 (5 'PLAG1; red signal) (Invitrogen, Carlsbad, CA).

\subsection{Comparative genomic hybridization on arrays (CGH-array) study}


Genomic DNA was extracted from FFPE tissues of the biopsy using a Promega Maxwell 16 FFPE kit (Promega, Madison, USA). Human reference DNA was provided by Agilent Technologies (Santa Clara, CA). Both DNAs were labeled using the Genomic DNA SureTag Labeling kit and co-hybridized onto a 4x180K SurePrint G3 Human CGH microarray (Agilent Technologies). The microarray was scanned using a SureScan scanner (Agilent Technologies). Data were analyzed using Agilent Cytogenomics software (version 2.9.2.4, Agilent Technologies) and expressed according to the hg19 reference genome (GRCh37, Genome Reference Consortium Human Build 37).

\subsection{Single-nucleotide polymorphism on arrays (SNP-array) study}

Genomic DNA was extracted from FFPE tissues of the surgical sample. Copy number alterations and loss of heterozygosity were evaluated using Affymetrix OncoScan CNV FFPE Assay with 80ng input of genomic DNA (Affymetrix, Santa Clara, CA). Experimental procedures were performed according to the manufacturer's recommendations (Affymetrix). Data were analyzed using the Chromosome Analysis Suite (ChAS 3.3) software (ThermoFisher Scientific, Waltham, MA). Annotations were based on the human reference hg19 (Genome Reference Consortium Human Build 37 (GRCh37)).

\subsection{Targeted next generation sequencing (targeted-NGS) analysis}

Targeted sequencing was performed using the Ion Torrent semiconductor-based sequencing technology (Life Technologies, Grand Island, NY). Libraries from the biopsy specimen were barcoded with a sample-specific 10nt-barcode sequence (Ion Xpress barcode adapter kit) and processed on a 318 v2 chip. Emulsion PCR (Ion One Touch 2, Thermo Fisher Scientific, Waltham, MA) was performed according to the manufacturer's recommendations. Sequencing was performed using the Ion Torrent Personal Genome Machine by targeting 50 genes with the Ion AmpliSeq Cancer Hotspots Panel v2. The data were analyzed using Torrent Suite software (version 5.6) and the Torrent Variant Caller plugin with the "somatic-low 
stringency" parameters. Variants were annotated using Ion Reporter software (version 5.0) and then expressed according to the hg19 reference genome (GRCh37, Genome Reference Consortium Human Build 37).

\section{RESULTS}

\subsection{Pathological findings}

Analysis of the biopsy specimen revealed a proliferation of mature adipocytes. No evidence of nuclear atypia was detected. Macroscopically, the complete surgical resection tumor specimen consisted of a yellowish, well-circumscribed and encapsulated monobloc measuring 10x8x7cm (Fig. 1B). No necrotic or hemorrhagic area was detected. Microscopically, a few large and hyperchromatic nuclei were observed within a proliferation of mature adipocytes mixed with thick fibrous septa (Fig. 1C-E). Immunostaining showed a strong nuclear expression of MDM2 (Fig. 1F) and HMGA2 (Fig. 1G).

\subsection{Cytogenetic and molecular findings}

MDM2 amplification was first detected in interphase cells from the biopsy sample. More than 20 extra copies per cell were observed (Fig. 2A). Strikingly, the signals were diffusely spread all over the nucleus, in contrast to the clusters usually observed in adult ALT/WDLPS/DDLPS (Fig. 2B). Both CGH-array and SNP-array analyses confirmed the amplification of MDM2 (mean $\mathrm{Cy} 5 / \mathrm{Cy} 3$ log ratio: 3.5; average copy number: 22). A large discontinuous 12q13-21 amplicon, was observed (from 53,036,089 up to 91,580,752 according to nucleotidic coordinates in human reference hg19 GRCh37). Overall, 64 genes were amplified (Supplementary Fig. 1). Notably, CDK4, FRS2 and CPM and a portion of HMGA2 (5' region) were co-amplified with $M D M 2$. This amplicon was surrounded by deleted regions at $12 \mathrm{q} 11-12$ and 12q22-24.3, respectively (Fig. 2E and Supplementary Fig. 1). The SNP-array results indicated an average of 23 copies of the MDM2 gene, and only two values (instead of the three 
expected) of Allele Difference: 2.997 and -2.997 and of B Allele Frequency (BAF): 0.003 and 0.99, respectively (Fig. 2F). This suggested a monoallelic origin of the amplified regions. The only other quantitative alteration detected was a loss of 10q11.21-q26.3 (Fig. 2E). FISH analysis on interphase cells from the surgical sample confirmed the $M D M 2$ amplification. No rearrangement of PLAG1 was detected. Only a few metaphase cells were obtained from the short-term cell culture. Even though the quality of R-banded cell metaphases was not sufficient to establish a full karyotype, it was possible to observe the presence of double minutes chromosomes (dmin) (data not shown). No giant marker or supernumerary ring chromosome was detected. FISH analysis showed that MDM2 amplification was located on dmin, as suspected from features observed on interphase nuclei (Fig. 2C-D). According to the International System for Human Cytogenomic Nomenclature (ISCN 2016) ${ }^{12}$, the molecular results were: ish dmin(MDM2amp). nuc ish (D12Z3x2,MDM2amp). $\operatorname{arr}[$ GRCh37]10q11.21q26.3(45374097_135434303)x1, 12q11q13.3(37902987_57838013)x1, 12q13.13q21.1(53036089_75664046)x22, 12q21.1q22(75682795_94826994)x1, 12q21.33(91317952_91580752)x21, 12q22q24.33(95364816_133818115)x1. No pathogenic mutation was detected using the NGS panel of 50 genes, including TP53 and PTEN.

\section{DISCUSSION}

In adults, the standard treatment of ALT/WDLPS consists in complete surgical resection of the tumor. ${ }^{2}$ In terms of prognosis, anatomic location is a major factor since tumors localized in the retroperitoneum are more prone to recurrence and dedifferentiation. Because ALT/WDLPS presents risks of recurrence, dedifferentiation and metastasis, a careful followup is mandatory. Considering the rarity of pediatric adipose tumors and the lack of hindsight regarding their clinical evolution, patient management can be difficult. The present description of a novel case of pediatric ALT confirms that malignant well-differentiated adipose tumors do 
occur in children. Checking of MDM2 status, even in the absence of evidence of nuclear atypia, is therefore highly recommended for any large or deep-seated or rapidly growing pediatric adipose tumor. Such MDM2 analysis is crucial to ensure an accurate diagnosis. Notably ALT may be misdiagnosed as lipoma in the absence of molecular analysis. According to the current World Health Organization classification of soft tissue and bone tumors, MDM2 amplification is a consistent distinctive feature separating WDLPS/ALT from benign adipocytic tumors, such as lipoma and lipoblastoma. ${ }^{2}$ An appropriate method for this detection is currently FISH analysis. Indeed, FISH allows a rapid quantitative determination of MDM2. It provides information on the level of MDM2 amplification in a large number of individual cells. Both immunohistochemistry and microarrays (CGH-array, SNP-array) may overlook MDM2 amplification: immunostaining for MDM2 is sometimes unreliable while microarray profiles can underestimate the level of MDM2 amplification in case of tumor heterogeneity. Indeed, if only a small proportion of cells of the tumor sample carry $M D M 2$ amplification, the apparent quantitative level of MDM2 may be low. Only three of the 25 pediatric WDLPS/ALT/DDLPS reported in the literature have been genetically studied using FISH analysis. ${ }^{3-5}$ MDM2 amplification was detected in one case of ALT from a 7-year-old girl ${ }^{3}$ while low-level amplification of $C D K 4$ and no amplification of MDM2 was found in an ALT in a 14-year-old girl. ${ }^{4}$ In the third case, a DDLPS occurring in an 8-year-old girl, Okamoto et al. did not detect MDM2 amplification. ${ }^{5}$ Our case and the sole other reported case of $M D M 2$-amplified pediatric ALT ${ }^{3}$ showed clinical and pathological differences: the case described by Peng et al $^{3}$ was a predominantly sclerosing deep-seated mass of the face while our case was a well-differentiated adipocytic tumor located in a limb. In the case described by Peng et al., the nucleic distribution of MDM2 signals was not discussed by the authors. In the present case, we performed CGHarray and SNP-array in addition to FISH analysis. This allowed confirmation of the high-level amplification of $M D M 2$ but also an overview of the quantitative profile of the whole tumor 
genome. A relatively simple genomic profile mainly characterized by $12 q 13-21$ amplification, similar to those observed in most adult WDLPS/ALT, was detected. In contrast, we noticed that the spatial configuration of $M D M 2$ on interphase nuclei of this pediatric tumor was different from the usual layout in clusters of MDM2 signals of adult tumors. These typical clusters result from the position of $12 \mathrm{q}$ amplicons on giant rod or ring chromosomes which are the cytogenetic mark of adult WDLPS/ALT/DDLPS. ${ }^{2,13}$ Only a few exceptions have been reported. For instance, in an adult case of DDLPS showing neither rings and large markers nor dmin, 12q amplification was scattered and inserted into several chromosomes. ${ }^{14}$ The presence of dmin, in addition to the classical ring or marker chromosomes, has been reported only in a few cases. ${ }^{15}$ FISH analysis was not always performed to identify the origin of these dmin. ${ }^{16-18}$ In four cases of adult WDLPS, the dmin were shown to contain sequences from chromosome 12. ${ }^{19,20}$ In contrast, in five other cases there was no evidence of sequences of chromosome 12 or $M D M 2$ in the dmin. ${ }^{17,19,21-23}$ In the present case, we observed that MDM2 signals were spread all over the nuclei. This prompted us to verify the chromosomal localization of the supernumerary MDM2 signals on metaphase cells. MDM2 amplification was located on dmin instead of being grouped within large marker or rings. Dmin are small paired acentric chromatin bodies. They are described as an extrachromosomal vector of gene amplification while homogeneously stained regions (hsr) represent the intrachromosomal carriers of amplification. ${ }^{24,25}$ In WDLPS/ALT/DDLPS, MDM2 amplification is carried by giant rods or ring chromosomes. Strikingly, whereas MDM2 owes its name to the initial discovery of amplification in mouse dmin ${ }^{26}$, the structure of MDM2 amplification in human has been the most deeply explored in giant rods or ring chromosomes of WDLPS/ALT/DDLPS. ${ }^{17,21,27-29}$ These odd chromosomes represent an original and very peculiar form of hsr since they are not inserted in a regular chromosome. They consist in supernumerary genomically complex structures harboring a neocentromere. ${ }^{21} M D M 2$ amplification has been observed in other 
tumors than WDLPS/ALT/DDLPS such as intimal sarcoma, osteosarcoma, glioma, breast cancer or salivary gland tumors. The chromosomal vector of MDM2 amplification has been scarcely studied in human tumors other than WDLPS/ALT/DDLPS. Dmin containing MDM2 have already been reported in salivary glands tumors and neuroblastoma. ${ }^{30,31}$ Our observation of MDM2 amplification carried by dmin in a pediatric case of adipose tumor raises the issue of the mechanism of their generation. The episome model of formation of dmin, which is based on excision of a gene from its original locus followed by amplification has been extensively studied in vitro. ${ }^{24,32}$ This model is not consistent with MDM2 amplification in ALT/WDLPS/DDLPS because MDM2 is usually not deleted on the pair of chromosomes 12 that remains in cells harboring rings and large markers. In the present case, MDM2 was not apparently deleted from chromosomes 12 (Fig. 2D). Mechanisms of recombination without deletion of original locus have also been described to produce dmin. ${ }^{33}$ However, these models are more compatible with amplification of a small region to select one or a couple of driver genes. It does not explain the generation of large amplicons such as those observed in ALT/WDLPS/DDLPS. Another model that may account for the formation of dmin in a variety of tumors is chromothripsis. ${ }^{34,35}$ According to Garsed et al. chromothripsis may be the initiating event in the formation of the ring chromosomes in ALT/WDLPS/DDLPS. ${ }^{27}$ Large scale sequencing of these chromosomes isolated from several WDLPS and DDLPS cell lines using flow cytometry was in favor of this mechanism rather than others, such as breakage-fusionbridge. Though not being directly the source of amplification, the major "catastrophic" rearrangement caused by chromothripsis phenomenon in $12 \mathrm{q}$ and in other chromosomes simultaneously may be the starting point of further copy number changes leading to amplification and to neocentromere formation and integration of telomeric sequences. In this model, the formation of dmin might directly follow the initial chromosome shattering and precede reassembly in rings and then, large marker chromosomes. In the present case, both the 
heterogeneity of dmin (a few of them did not contain MDM2: see Fig. 2D) and the SNP-array profile indicating an alternation of $\mathrm{LOH}$, monoallelic amplification and preserved regions could be in favor of a chromothripsis mechanism of formation of dmin chromosomes. ${ }^{35,36}$

Our findings of dmin bearing MDM2 amplification in a pediatric case raise the issue of the potential evolution of these dmin if the tumor had not been surgically removed. We cannot predict whether these dmin would have aggregated leading to ring and large markers similar to those observed in most adult cases of WDLPS/ALT/DDLPS. Former observations in neuroblastomas suggested that dmin containing $M Y C N$ and $M D M 2$ could evolve towards hsr in human tumors. ${ }^{31}$ Similar hypotheses have been raised in lung cancer cell lines. ${ }^{37}$ However, in vivo proof of such an aggregation of dmin resulting in hsr in primary tumors is missing. Alternatively, we cannot exclude the possibility that they would have remained as dmin and constitute a distinct entity from adult cases. Prospective exhaustive genetic studies of these rare pediatric lipomatous tumors may help resolve these issues.

Acknowledgments: The authors are thankful to Roger Grattery, Annie-Claude Peyron, Frédérique Keslair and Audrey Bazin for their technical assistance and Jean-François Michiels and Zoé Pedeutour-Braccini for their pathological evaluation. 


\section{References}

1. Dadone B, Refae S, Lemarié-Delaunay C, Bianchini L, Pedeutour F. Molecular cytogenetics of pediatric adipocytic tumors. Cancer Genet. 2015;208(10):469-481.

2. Dei Tos AP, Pedeutour F. Atypical lipomatous tumour. In: Fletcher DM, Bridge JA, Hogendoorn PCW, Mertens F, editors. WHO Classification of Tumours of Soft Tissue and Bone. $4^{\text {th }}$ ed. Lyon: IARC; 2013, p. 33-6.

3. Peng R, Chen H, Yang X, et al. A novel sclerosing atypical lipomatous tumor/welldifferentiated liposarcoma in a 7-year-old girl: report of a case with molecular confirmation. Hum Pathol. 2018;71:41-46.

4. Kuhnen C, Mentzel T, Fisseler-Eckhoff A, Debiec-Rychter M, Sciot R. Atypical lipomatous tumor in a 14-year-old patient: distinction from lipoblastoma using FISH analysis. Virchows Arch Int J Pathol. 2002;441(3):299-302.

5. Okamoto S, Machinami R, Tanizawa T, Matsumoto S, Lee G-H, Ishikawa Y. Dedifferentiated liposarcoma with rhabdomyoblastic differentiation in an 8-year-old girl. Pathol Res Pract. 2010;206(3):191-196.

6. Huh WW, Yuen C, Munsell M, et al. Liposarcoma in children and young adults: a multiinstitutional experience. Pediatr Blood Cancer. 2011;57(7):1142-1146.

7. Alaggio R, Coffin CM, Weiss SW, et al. Liposarcomas in young patients: a study of 82 cases occurring in patients younger than 22 years of age. Am J Surg Pathol. 2009;33(5):645658.

8. Debelenko LV, Perez-Atayde AR, Dubois SG, et al. p53+/mdm2- atypical lipomatous tumor/well-differentiated liposarcoma in young children: an early expression of Li-Fraumeni syndrome. Pediatr Dev Pathol Off J Soc Pediatr Pathol Paediatr Pathol Soc. 2010;13(3):218224.

9. Stanelle EJ, Christison-Lagay ER, Sidebotham EL, et al. Prognostic factors and survival in pediatric and adolescent liposarcoma. Sarcoma. 2012;2012:870910.

10. Hahn HP, Fletcher CDM. Primary mediastinal liposarcoma: clinicopathologic analysis of 24 cases. Am J Surg Pathol. 2007;31(12):1868-1874.

11. Boland JM, Colby TV, Folpe AL. Liposarcomas of the mediastinum and thorax: a clinicopathologic and molecular cytogenetic study of 24 cases, emphasizing unusual and diverse histologic features. Am J Surg Pathol. 2012;36(9):1395-1403.

12. McGowan-Jordan J, Simons A, Schmid M, eds. An International System for Human Cytogenomic Nomenclature. Basel, Switzerland: Karger; 2016.

13. Dei Tos AP, Marino-Enriquez A, Pedeutour F, Rossi S. Dedifferentiated liposarcoma. In: Fletcher DM, Bridge JA, Hogendoorn PCW, Mertens F, editors. WHO Classification of Tumours of Soft Tissue and Bone. 4th ed. Lyon: IARC; :37-38.

14. Mandahl N, Magnusson L, Nilsson J, et al. Scattered genomic amplification in dedifferentiated liposarcoma. Mol Cytogenet. 2017;10:25.

15. Mitelman Database of Chromosome Aberrations and Gene Fusions in Cancer (2019). Mitelman F, Johansson B and Mertens F http://cgap.nci.nih.gov/Chromosomes/Mitelman. Accessed February 26, 2019.

16. Bassett MD, Schuetze SM, Disteche C, et al. Deep-seated, well differentiated lipomatous tumors of the chest wall and extremities: the role of cytogenetics in classification and prognostication. Cancer. 2005;103(2):409-416.

17. Gisselsson D, Höglund M, Mertens F, et al. The structure and dynamics of ring chromosomes in human neoplastic and non-neoplastic cells. Hum Genet. 1999;104(4):315-325. 18. Sreekantaiah C, Karakousis CP, Leong SP, Sandberg AA. Cytogenetic findings in liposarcoma correlate with histopathologic subtypes. Cancer. 1992;69(10):2484-2495. 
19. Sozzi G, Minoletti F, Miozzo M, et al. Relevance of cytogenetic and fluorescent in situ hybridization analyses in the clinical assessment of soft tissue sarcoma. Hum Pathol. 1997;28(2):134-142.

20. Pilotti S, Della Torre G, Mezzelani A, et al. The expression of MDM2/CDK4 gene product in the differential diagnosis of well differentiated liposarcoma and large deep-seated lipoma. Br J Cancer. 2000;82(7):1271-1275.

21. Pedeutour F, Suijkerbuijk RF, Forus A, et al. Complex composition and coamplification of SAS and MDM2 in ring and giant rod marker chromosomes in welldifferentiated liposarcoma. Genes Chromosomes Cancer. 1994;10(2):85-94.

22. Dal Cin P, Kools P, Sciot R, et al. Cytogenetic and fluorescence in situ hybridization investigation of ring chromosomes characterizing a specific pathologic subgroup of adipose tissue tumors. Cancer Genet Cytogenet. 1993;68(2):85-90.

23. Nilsson M, Meza-Zepeda LA, Mertens F, Forus A, Myklebost O, Mandahl N. Amplification of chromosome 1 sequences in lipomatous tumors and other sarcomas. Int $J$ Cancer. 2004;109(3):363-369.

24. Storlazzi CT, Lonoce A, Guastadisegni MC, et al. Gene amplification as double minutes or homogeneously staining regions in solid tumors: origin and structure. Genome Res. 2010;20(9):1198-1206.

25. Turner KM, Deshpande V, Beyter D, et al. Extrachromosomal oncogene amplification drives tumour evolution and genetic heterogeneity. Nature. 2017;543(7643):122-125.

26. Fakharzadeh SS, Trusko SP, George DL. Tumorigenic potential associated with enhanced expression of a gene that is amplified in a mouse tumor cell line. EMBO J. 1991;10(6):1565-1569.

27. Garsed DW, Marshall OJ, Corbin VDA, et al. The architecture and evolution of cancer neochromosomes. Cancer Cell. 2014;26(5):653-667.

28. Pedeutour F, Forus A, Coindre JM, et al. Structure of the supernumerary ring and giant rod chromosomes in adipose tissue tumors. Genes Chromosomes Cancer. 1999;24(1):30-41.

29. Sirvent N, Forus A, Lescaut W, et al. Characterization of centromere alterations in liposarcomas. Genes Chromosomes Cancer. 2000;29(2):117-129.

30. Rao PH, Murty VV, Louie DC, Chaganti RS. Nonsyntenic amplification of MYC with CDK4 and MDM2 in a malignant mixed tumor of salivary gland. Cancer Genet Cytogenet. 1998;105(2):160-163.

31. Corvi R, Savelyeva L, Amler L, Handgretinger R, Schwab M. Cytogenetic evolution of MYCN and MDM2 amplification in the neuroblastoma LS tumour and its cell line. Eur $J$ Cancer Oxf Engl 1990. 1995;31A(4):520-523.

32. Wahl GM. The importance of circular DNA in mammalian gene amplification. Cancer Res. 1989;49(6):1333-1340.

33. Stark GR. Regulation and mechanisms of mammalian gene amplification. Adv Cancer Res. 1993;61:87-113.

34. Stephens PJ, Greenman CD, Fu B, et al. Massive genomic rearrangement acquired in a single catastrophic event during cancer development. Cell. 2011;144(1):27-40.

35. Rode A, Maass KK, Willmund KV, Lichter P, Ernst A. Chromothripsis in cancer cells: An update. Int J Cancer. 2016;138(10):2322-2333.

36. Zhang C-Z, Leibowitz ML, Pellman D. Chromothripsis and beyond: rapid genome evolution from complex chromosomal rearrangements. Genes Dev. 2013;27(23):2513-2530.

37. Fukumoto M, Suzuki A, Inazawa J, et al. Chromosomal location and structure of amplicons in two human cell lines with coamplification of c-myc and Ki-ras oncogenes. Somat Cell Mol Genet. 1993;19(1):21-28. 


\section{FIGURE LEGENDS}

Figure 1. Radiological and pathological features. A. T1-weighted, axial section of Magnetic Resonance Imagery with fat saturation sequences showing an extinction of the hypersignal of the adipocytic tumor (arrow). The tumor was deep-seated in the adductor muscle. B. Gross aspect of the resection: encapsulated and adipose tumor without necrosis or hemorrhage. $\mathbf{C}$. Histologically, the tumor was composed of a proliferation of mature adipocytes with thick fibrous septa. D. High power view of the fibrous septa showing some striated muscle fibers (arrow). E. High power view of the mature adipocytes showing a few atypia with hyperchromatic and large nuclei. Immunohistochemical staining showing strong nuclear expression of MDM2 (F) and HMGA2 (G).

Figure 2. Genetics features. A-D. Fluorescence In Situ Hybridization (FISH) analyses using probes for MDM2 (green signals) and centromere 12 (red signals). Interphase nuclei and metaphase chromosomes are counterstained in blue (DAPI). A. In interphase nuclei, the two red signals indicated the presence of two centromeres of chromosome 12. The MDM2 amplification was diffuse, homogeneously distributed all over the nuclei; this distribution suggested the presence of double minute (dmin) chromosomes. B. Example of a classical cluster of MDM2 amplified signals in an adult's atypical lipomatous tumor. C. Yellow circles focus on numerous dmin chromosomes present in a few metaphase cells of the pediatric ALT. D. Results of FISH analysis demonstrated that the MDM2 amplification was carried by the dmin chromosomes; Two chromosome 12 are indicated by white arrows; Orange arrows indicated few dmin without MDM2 signals. E. Quantitative whole genome profile representation of Single Nucleotide Polymorphism (SNP) array analysis showing high-level large and discontinuous amplicon surrounded by a loss of 12q11-12 and 12q22-24.3 regions (blue 
arrowhead) and a loss of the long arm of chromosome 10 (black arrowhead). F. SNP-array results showing a focus on the $12 \mathrm{q}$ region (genomic coordinates from 64.470 .744 to 73.970.757). The figure was centered by the MDM2 gene (black arrowhead). The red bars represented losses while the blue bar, gains or amplification. The Allele Difference and the B Allele Frequency represented the variation of the heterozygosity.

Supplementary Figure 1. Genes amplified in the 12q amplicons. We observed discontinuous amplicons located on 12q13.13-15 and on 12q21.1-21.33, including several genes and notably MDM2, CDK4, HMGA2, CPM, FRS2 and YEATS4. 

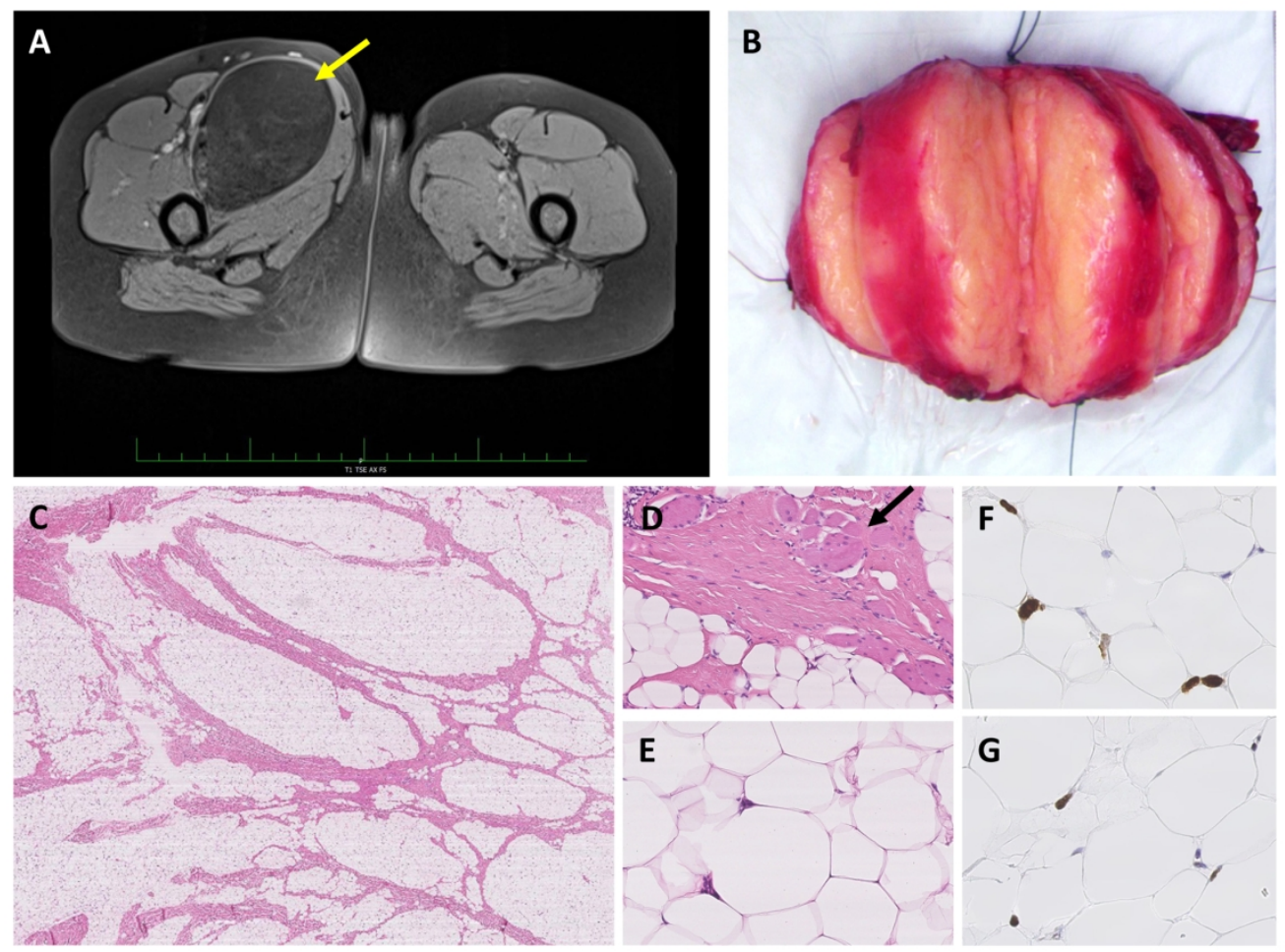

Figure 1. Radiological and pathological features. A. T1-weighted, axial section of Magnetic Resonance Imagery with fat saturation sequences showing an extinction of the hypersignal of the adipocytic tumor (arrow). The tumor was deep-seated in the adductor muscle. B. Gross aspect of the resection: encapsulated and adipose tumor without necrosis or hemorrhage. C. Histologically, the tumor was composed of a proliferation of mature adipocytes with thick fibrous septa. D. High power view of the fibrous septa showing some striated muscle fibers (arrow). E. High power view of the mature adipocytes showing a few atypia with hyperchromatic and large nuclei. Immunohistochemical staining showing strong nuclear expression of MDM2 (F) and HMGA2 (G). 

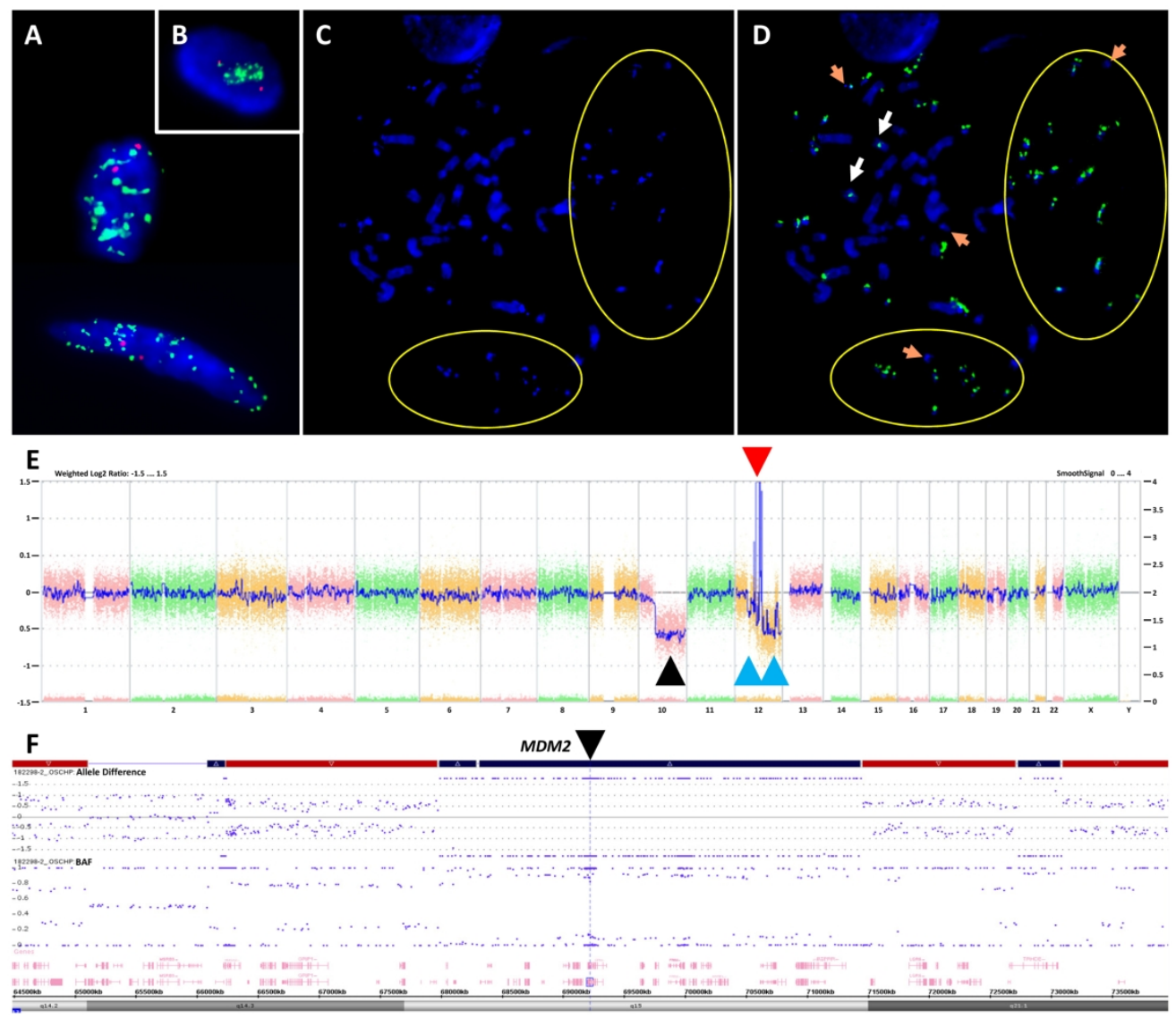

Figure 2. Genetics features. A-D. Fluorescence In Situ Hybridization (FISH) analyses using probes for MDM2 (green signals) and centromere 12 (red signals). Interphase nuclei and metaphase chromosomes are counterstained in blue (DAPI). A. In interphase nuclei, the two red signals indicated the presence of two centromeres of chromosome 12. The MDM2 amplification was diffuse, homogeneously distributed all over the nuclei; this distribution suggested the presence of double minute (dmin) chromosomes. B. Example of a classical cluster of MDM2 amplified signals in an adult's atypical lipomatous tumor. C. Yellow circles focus on numerous dmin chromosomes present in a few metaphase cells of the pediatric ALT. D. Results of FISH analysis demonstrated that the MDM2 amplification was carried by the dmin chromosomes; Two chromosome 12 are indicated by white arrows; Orange arrows indicated few dmin without MDM2 signals. E. Quantitative whole genome profile representation of Single Nucleotide Polymorphism (SNP) array analysis showing high-level large and discontinuous amplicon surrounded by a loss of 12q11-12 and 12q22-24.3 regions (blue arrowhead) and a loss of the long arm of chromosome 10 (black arrowhead). F. SNP-array results showing a focus on the $12 \mathrm{q}$ region (genomic coordinates from 64.470.744 to 73.970.757). The figure was centered by the MDM2 gene (black arrowhead). The red bars represented losses while the blue bar, gains or amplification. The Allele Difference and the B Allele Frequency represented the variation of the heterozygosity. 


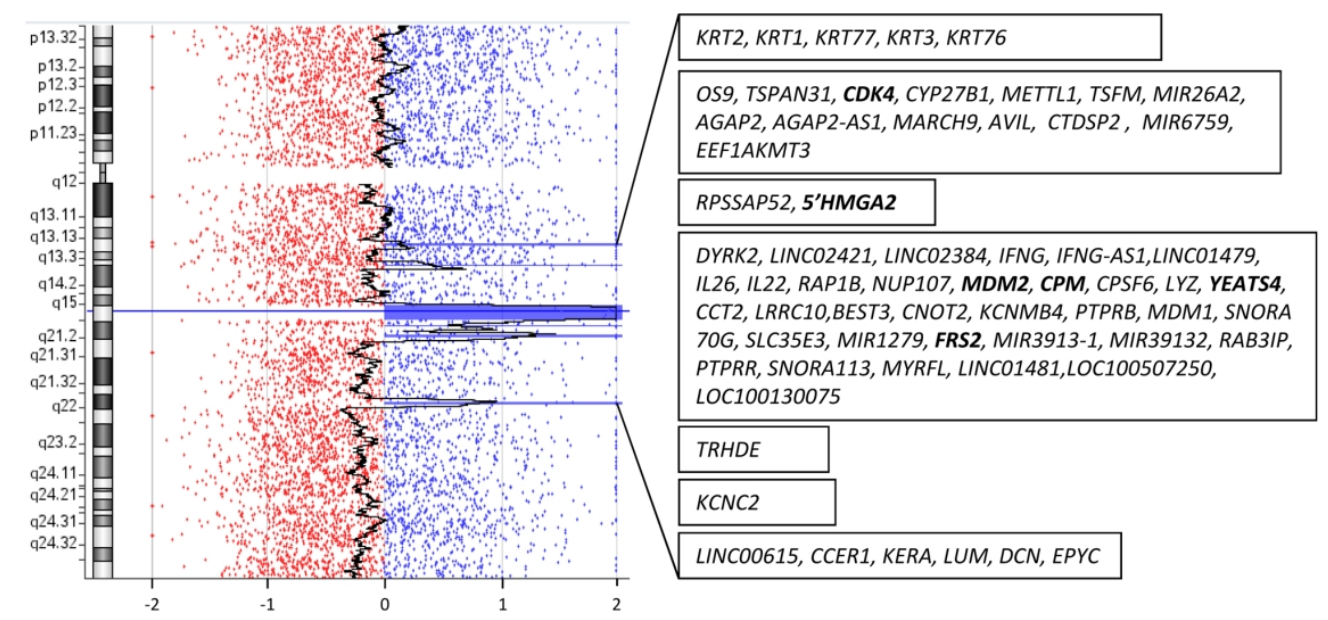

